\title{
Flora vascular y vegetación del humedal de Santa Rosa (Chancay, Lima) Vascular flora and vegetation of Santa Rosa wetland (Chancay, Lima)
}

\author{
Damaso W. Ramirez ${ }^{1}$, Hector Aponte ${ }^{1}$ y Asuncion Cano ${ }^{1,2}$
}

1 Museo de Historia Natural - Laboratorio de Florística. Universidad orion Acional Mayor de San Marcos. Av. Arenales 1256 Jesús María. LimaPerú. Email Damaso Ramirez: wilsonxviii@Gmail.com

2 Instituto de Investigación de Ciencias Biológicas Antonio Raimondi (ICBAR), Facultad de Cimond (ICBAR), Facultad de Ciencias Biologicas, Universidad Mayor de San Marcos.

Trabajo presentado a la XVIII Reunión Científica del Instituto de Investigaciones en Ciencias Biológicas Antonio Raimondi, "200 años gicas Antonio Raimondi, "200 años
del nacimiento de Charles Darwin y del nacimiento de Charles Darwin y el 150 aniversario de la publicación
de On the Origin of Species by Means of Natural Selection". Del 19 al 21 de agosto de 2009 .

Publicado impreso: 20/10/2010 Publicado online: 29/09/2010

\section{Resumen}

El presente trabajo muestra los resultados de un estudio de la flora y vegetación del humedal de Santa Rosa (Chancay, Lima) realizado entre los años 2007 y 2009. La flora vascular estuvo conformada por 66 especies agrupadas en 57 géneros y 26 familias. Las Poaceae (16), Cyperaceae (6) y Asteraceae (6) fueron las familias más diversas y constituyeron el $42 \%$ de la flora total. En comparación con los principales humedales de Lima, Santa Rosa presenta un mayor número de especies (Pantanos de Villa (65), Paraíso (26) y Medio Mundo (16)). El análisis de similitud florística muestra que Santa Rosa es más semejante a Los Pantanos de Villa (Lima). El Análisis de Correspondencia (AC) determinó tres comunidades vegetales predominantes: a) Comunidad de acuáticas flotantes, b) Totoral y asociadas y c) Vega Mixta. Se considera que los cambios en la estructura de las comunidades vegetales y el número de especies introducidas son indicadores del impacto antrópico al que está sometido este ecosistema. Se identifican las fuentes de impacto humano y se considera a la agricultura como la principal amenaza del humedal.

Palabras clave: Acuáticas, Antropización, desierto costero, Agricultura, Perú.

\section{Abstract}

The present work shows the results of flora and vegetation study made between 2007 and 2009 on Santa Rosa wetland (Chancay - Lima). The vascular flora was composed for 66 species, grouped on 57 genus and 26 families. The Poaceae (16), Cyperaceae (6) and Asteraceae (6) are the more diverse families and represent the $42 \%$ of the flora. A comparison between the principal wetlands of Lima shows that Santa Rosa has more plant species (Pantanos de Villa (65), Paraíso (26) and Medio Mundo (16)). The analysis of floristic similarity between those localities shows that Santa Rosa is more similar to Pantanos de Villa (Lima) than the other localities. Using a Correspondence Analysis (CA), it was possible to recognize just three kinds of plant communities: a) Floating aquatic plants community, b) Totoral with associated species and c) Mixed Vega. It is considered that the changes on the plant community structure and the quantity of invasive species are indicators of the anthropic impact on the area. Human impact sources were identified and agriculture is considered the most important menace of this ecosystem.

Keywords: Aquatics, anthropization, coastal desert, agriculture, Peru.

\section{Introducción}

Los humedales son ecosistemas en constante relación con masas de agua donde se encuentran plantas adaptadas a estas condiciones (Mitsch \& Gosselink 1993). Estos ecosistemas son considerados entre los más importantes del mundo por las funciones medio ambientales que realizan, como controlar los cursos de las corrientes de agua, participar en la regulación del carbono global, proporcionar hábitat a centenares de especies animales y vegetales, y poseer un importante valor cultural y recreacional (Clarkson et al. 2004). Comprender cómo estos ecosistemas han evolucionado frente al impacto humano ha sido objeto de múltiples investigaciones (Young 1998, USDA 2000, Christophenson 1994, Clarkson et al. 2004, Cooke et al. 1990).

Los humedales de Lima son parte de un corredor biológico a lo largo del desierto costero del Perú. La mayor parte de estos ambientes tienen recursos que son explotados, como por ejemplo el junco Schoenoplectus americanus que es utilizado para la fabricación de diversas artesanías (León \& Young 1996, León et al. 1998). La coexistencia de los humedales con las poblaciones humanas ocasionan su deterioro, tanto por las actividades que se realizan en ellos como por la contaminación (vertimiento de efluentes domésticos, depósitos de basura) (Young 1998). Varias investigaciones acerca de estos ecosistemas costeros han sido realizados (Cano et al. 1998, León et al. 1995, León et al. 1997, Montoya 1998, Arana \& Salinas 2003), sin embargo, estudios de las comunidades vegetales y del estado de conservación en los humedales no protegidos son escasos.

El presente trabajo estudia la flora y la composición de las comunidades vegetales del humedal de Santa Rosa, uno de los humedales no protegidos, ubicado al norte de la ciudad de Lima.

\section{Área de estudio}

El humedal de Santa Rosa (11³6’01,4”S - 77¹5’54,0”W) se encuentra al norte de la ciudad de Lima, Provincia de Huaral, distrito de Chancay (Fig. 1); tiene un área aproximada de 32 hectáreas, con un cuerpo de agua en la zona central, una pe-

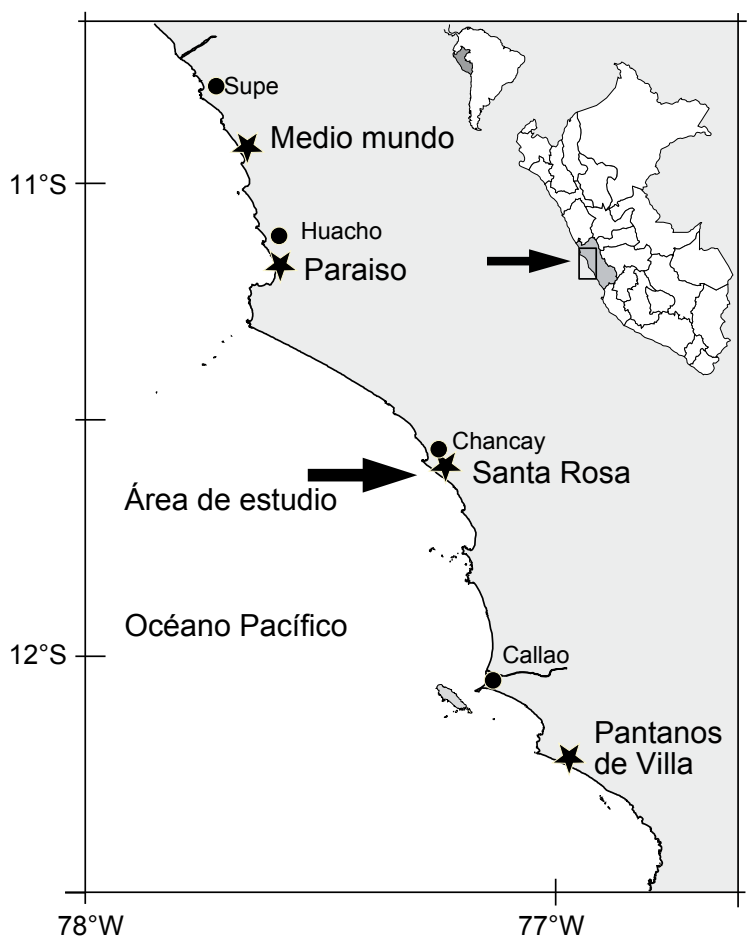

Figura 1. Mapa del área de estudio, Humedal de Santa Rosa. Se indica la ubicación de los humedales costeros de Paraíso, Medio Mundo, Pantanos de Villa y Santa Rosa. 
queña laguna al lado oeste y un canal principal que lo abastece de agua, proveniente del rió Chancay. El humedal se encuentra rodeado por varias fuentes de impacto antropogénico. Al sur (Peralvillo y Salinas Alta) se encuentran campos de cultivo de algodón, camote, fresas y zapallo. Al oeste podemos encontrar botaderos de basura y criaderos de cerdos. Al este y norte se encuentran la zona urbana de Santa Rosa. La zona este del humedal es utilizada frecuentemente como área de pastoreo ovino y vacuno.

\section{Materiales y métodos Estudio de la flora del humedal}

Entre marzo del 2007 y enero del 2009, fueron realizadas colectas en los diferentes hábitats del humedal utilizando técnicas estandarizadas (Bridson \& Forman 1992). Se registró la forma de crecimiento (Wittaker 1975).

Las determinaciones taxonómicas fueron realizadas en el Laboratorio de Florística del Museo de Historia Natural de la Universidad Nacional Mayor de San Marcos, empleando claves y descripciones publicadas en literatura especializada (Sagastegui 1973, León 1993, Tovar 1993), además de consultas a especialistas. Las muestras fueron contrastadas con especímenes depositados en el Herbario San Marcos (USM). Se consideró como especies introducidas a todas aquellas provenientes de otros habitas que no estén naturalizadas, e invasoras a los provenientes de los campos de cultivos. Los ejemplares colectados fueron depositados en el Herbario San Marcos (USM).

\section{Estudio de las comunidades vegetales del humedal}

Con la finalidad de determinar la composición y dominancia de las distintas comunidades vegetales del humedal, se realizaron un total de 15 transectos en enero del 2009. Los transectos se ubicaron de forma sistemática sobre las comunidades vegetales del humedal, identificadas por su fisonomía (Cano et al. 1998). En cada transecto se aplicó el método de intersección de puntos modificado (BOLFOR et al. 2000); que caracteriza la vegetación a partir de mediciones de cobertura y es adecuado para comunidades vegetales donde es difícil discriminar individuos (hierbas, pastizales, bofedales, césped de puna). Cada transecto tuvo una longitud de $10 \mathrm{~m}$. Se tomo un total de 50 puntos por transecto, en cada punto se registraron las especies que tocaron una varilla de $1,5 \mathrm{~m}$. Asimismo se anotó la cantidad de veces que cada especie era tocada por una varilla a fin de obtener una idea de la cobertura total en el punto y la sumatoria de los 50 puntos se consideró como su medida de cobertura. Si no se encontraba nada en ese punto se consideró vacío.

Los datos obtenidos de la evaluación de transectos fueron evaluados mediante un análisis de correspondencia (CA) utilizando el software PAST 1.89 (Hammer et al. 2001).

\section{Comparación de la flora entre humedales}

Se construyó una matriz de presencia-ausencia de las especies

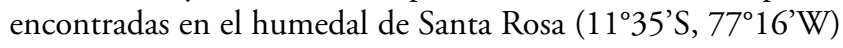
para compararla con otros humedales de la costa central Pantanos de Villa (Lima, $\left.12^{\circ} 11^{\prime} \mathrm{S}, 7^{\circ} 59^{\prime} \mathrm{W}\right)$; la laguna El Paraíso (Huacho, $11^{\circ} 11^{\prime} \mathrm{S}, 77^{\circ} 35^{\prime} \mathrm{W}$ ) y la albufera de Medio Mundo (Vegueta, $10^{\circ} 55^{\prime} \mathrm{S}, 77^{\circ} 40^{\prime} \mathrm{W}$ ) (Cano et al. 1998). Con estos datos se realizó un análisis de agrupamiento haciendo uso del índice de Jaccard mediante el software PAST 1.89 (Hammer et al. 2001).

\section{Resultados \\ Flora del humedal de Santa Rosa}

Fueron determinadas un total de 66 especies, agrupadas en 57 géneros y 26 familias (Apéndice 1). Las Liliopsidas (Monocotiledóneas) representaron el 53\% de las especies, las Magnoliopsidas (Dicotiledóneas) el 44\% y los Pteridófitos el 3\%. Las familias con mayor número de especies fueron Poaceae (16), Cyperaceae (6) y Asteraceae (6) que constituyen el 42\% de la flora. Cinco familias (Apiaceae, Chenopodiaceae, Lemnaceae, Onagraceae y Polygonaceae) contienen tres especies cada una, otras cuatro (Aizoaceae, Araceae, Lamiaceae y Verbenaceae) presentan dos y el resto de familias presentan una especie cada una. Es importante mencionar que el 50\% (33 especies) de la flora total fueron consideradas como introducidas.

La forma de crecimiento predominante fueron las hierbas, las cuales constituyeron el 96\% de la flora total (63 especies), los arbustos sólo representaron el 4\% (3 especies), las hierbas acuáticas flotantes están representadas con 6 especies, las emergentes y las sumergidas con 2 y 1 especie respectivamente.

\section{Análisis de comunidades}

El análisis de correspondencia (CA) permitió identificar tres tipos de comunidades vegetales presentes en el humedal de Santa Rosa (Fig. 2):

Comunidad de acuáticas flotantes: Esta comunidad está asociada a gran parte de los espejos de agua y canales de la zona; la especie predominante es el repollito de agua Pistia stratiotes, acompañado de Hydrocotyle ranunculoides, que es una hierba arrigida emergente y raramente asociada con otras especies. Mediante observaciones de campo, se pudo apreciar que esta comunidad se encuentra asociada a Eichhornia crassipes que forma pequeños camalotales junto con esta comunidad.

Totoral y especies asociadas: Esta comunidad se desarrolla en sustratos inundados hacia el lado sur oeste del humedal. Las especies predominantes son Typha domingensis y Enhydra sessilifolia, ambas especies emergentes y en los alrededores encontramos Eichhornia crassipes una flotante libre. Una especie emergente (Myriophyllum aquaticum) tuvo una presencia importante en esta comunidad al inicio del estudio, sin embargo, fue escasa al momento de hacer los transectos.

Vega mixta: Esta comunidad se desarrolla en suelos pantanosos contiguos a los cuerpos de agua y se caracteriza por su porte bajo, se encuentran asociadas a zonas de pastoreo. Esta compuesta por múltiples especies entre las que predominan las ciperáceas (Cyperus laevigatus, Eleocharis geniculata, Schoenoplectus americanus), especies anfibias (Baccopa monnieri, Hydrocotyle ranunculoides, Paspalum vaginatum), y especies del gramadal (Distichlis spicata, Sarcocornia nei). También se encuentran gran parte de las especies consideradas como introducidas (Apéndice 1) entre las que se encuentran Medicago lupulina, Oxalis corniculatra y Eleusine indica. De igual manera hacia el borde de esta comunidad es posible apreciar el crecimiento de Colocasia esculenta y Tessaria integrifolia (pájaro bobo) que forma pequeños grupos y representaría una zona arbustiva relicto a lado del canal principal en el límite sur este. 


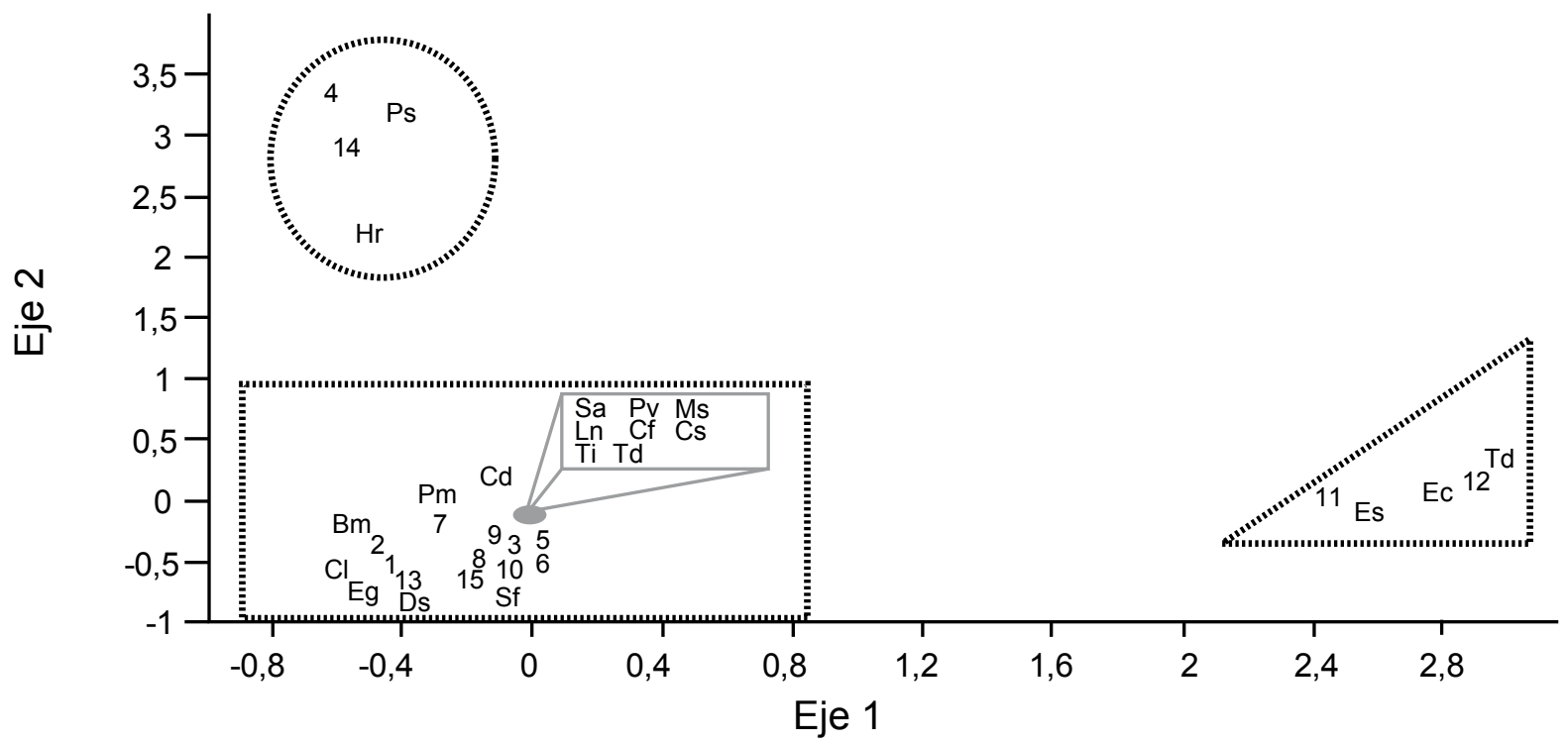

Figura 2. Comunidades obtenidas mediante el análisis de correspondencia (AC) utilizando el software PAST 1.89. EC=Eichornia crassipes; Es=Enydra sessiflora; Td=Typha domingensis; Ps=Pistia stratiotes; $\mathrm{Bm}=$ Baccopa monnieri; Sa=Schoenoplectus americanus; $\mathrm{Cd}=C \mathrm{Cynodon}$ dactylon; $\mathrm{Ds}=$ Distichlis spicata; $\mathrm{Sf}=$ Sarcocornia nei; $\mathrm{Pv}=$ Paspalum vaginatum; $\mathrm{Ms}=$ Mentha spicata; $\mathrm{Cl}=$ Cyperus laevigatus; $\mathrm{Eg}=$ Eleocharis geniculata; $\mathrm{Hr}=H y d r o c o t y l e$ ranunculoides; $\mathrm{Ln}=$ Lippia nodiflora; $\mathrm{Pm}=$ Plantago major; $\mathrm{Cf}=$ Conmelinna fasciculata; Cs=Colocassia esculenta; $\mathrm{Ti}=$ Tessaria integrifolia. Los números del 1 al 15 corresponden a los transectos evaluados.

\section{Comparación de la flora entre humedales}

La Tabla 1 muestra un cuadro comparativo entre los humedales de Santa Rosa, Pantanos de Villa, Paraíso y Medio Mundo. Las cuatro localidades presentan un patrón similar en la composición de familias, siendo las predominantes las Poáceas Cyperáceas y Asteráceas. Los dos humedales de mayor cantidad de taxones son el humedal de Santa Rosa y los Pantanos de Villa.

La mayor similitud se observa entre Santa Rosa y los Pantanos de Villa (34\%, índice de Jaccard, Fig. 3) compartiendo 34 especies, que corresponde al 51\% del total de especies registradas en el humedal de Santa Rosa (Tabla 1). Menor relación se encontró entre el humedal de Santa Rosa y los humedales de Paraíso y Medio Mundo, los cuales compartieron 16 y 9 especies respectivamente con el humedal de Santa Rosa (Apéndice 1). Estas dos últimas localidades forman un grupo aparte con un $54 \%$ de similitud y comparten un total de 13 especies.

\section{Discusión}

La flora vascular de Santa Rosa presenta un patrón similar a los otros humedales costeros en relación a las familias más dominan-

Tabla 1. Comparación del número de especies de las principales familias en los humedales de Santa Rosa, Pantanos de Villa, Paraíso y Medio Mundo. 'Basado en Cano, et al. (1998) corregido.

\begin{tabular}{lcccc}
\hline Familias & $\begin{array}{c}\text { Santa } \\
\text { Rosa }\end{array}$ & $\begin{array}{c}\text { Pantanos }_{\text {de Villa }^{\mathbf{1}}} \\
\text { Paraíso }^{\mathbf{1}}\end{array}$ & $\begin{array}{c}\text { Medio } \\
\text { Mundo }^{\mathbf{1}}\end{array}$ \\
\hline Poaceae & 16 & 9 & 7 & 3 \\
Cyperaceae & 6 & 8 & 4 & 4 \\
Asteraceae & 6 & 6 & 4 & 0 \\
Apiaceae & 3 & 3 & 0 & 0 \\
Chenopodiaceae & 3 & 2 & 2 & 2 \\
Lemmnaceae & 3 & 4 & 0 & 0 \\
Onagraceae & 3 & 4 & 0 & 0 \\
Poligonaceae & 3 & 1 & 0 & 0 \\
Resto de Familias & 23 & 28 & 8 & 7 \\
\hline Total & $\mathbf{6 6}$ & $\mathbf{6 5}$ & $\mathbf{2 5}$ & $\mathbf{1 6}$ \\
\hline
\end{tabular}

tes; Poaceae, Cyperaceae, y Asteraceae. Santa Rosa actualmente presenta un número de especies elevado tomando en cuenta el área que ocupa es menor que los otros humedales estudiados; Pantanos de Villa, Medio Mundo, y Paraíso. Sin embargo, esto no indica que sea el mas diverso en cuanto a flora nativa de humedales, ya que varias de las especies presentes en este humedal fueron consideradas invasoras de cultivo tales como; Chenopodium ambrossoides, Killinga brevifolia, Echinochloa cruspavonis, Echinochloa oryzoides, Eleusine indica, Rumex obtusifolius, entre otras. La presión antrópica sobre este humedal puede asociarse a la elevada proporción de especies introducidas (50\%).

Trabajos previos en Pantanos de Villa (Lima) señalan formaciones vegetales como el totoral, vega, gramadal, acuáticas y zona arbustiva (Young 1998; Cano et al 1998). En el humedal de Santa Rosa solo se identificaron tres formaciones vegetales (acuáticas flotantes, totoral, y vega mixta), lo cual indica una reestructuración de las comunidades originalmente presentes. La reducción de formaciones como el gramadal y vega dan origen a lo que llamamos una vega mixta la cual es consecuencia directa del impacto agrícola y ganadero. El pastoreo impide que las

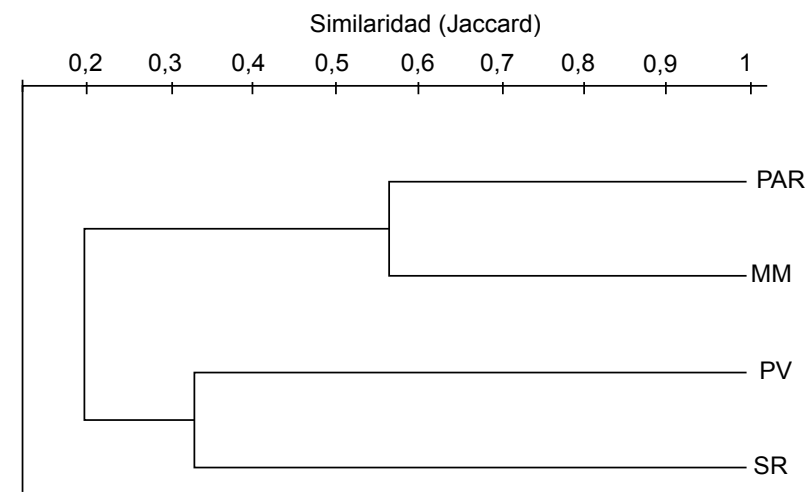

Figura 3. Dendograma de similitud entre la flora de los humedales costeros de Paraíso (PAR), Medio Mundo (MM), Pantanos de Villa (PV) y Santa Rosa (SR) a partir de una matriz de presencia / ausencia del total de las especies reportadas. 
especies dominantes (p.e. Schoenoplectus americanus) ocupen su extensión potencial, creando una competencia determinada por la reproducción clonal. La estructura en macollos de Eleocharis geniculata y Cyperus laevigatus es resistente y permite regenerarse y competir frente al ramoneo. Las especies acuáticas aprovechan los espacios vacíos dejados por el consumo ganadero y despliegan su hábito de corto tamaño y de extensión clonal. Igualmente, las especies oportunistas e invasoras ocupan estos nichos potenciales.

La actividad agrícola no solo ocasiona la presencia de especies invasoras en el humedal, también afecta a la comunidad de plantas acuáticas, probablemente aportando nutrientes a la laguna, los cuales llegarían por escorrentía desde los campos de cultivo adyacentes, cuando estos son fertilizados y regados, causando el incremento de las poblaciones de especies acuáticas como; Pistia stratiotes, Eichhornia crassipes y Lemna gibba los cuales actualmente destacan por la alta densidad poblacional cubriendo gran parte de los cuerpo de agua del humedal. Este fenómeno no se presenta en los otros humedales costeros mencionados.

El presente estudio muestra que la composición florística y la estructuración de comunidades son indicadores que deben considerarse dentro de los planes de manejo y conservación de áreas naturales como esta.

\section{Agradecimientos}

Agradecemos a Efraín Arana por su ayuda en las colectas iníciales. Queremos agradecer a María I. La Torre, Marybel Morales y Jorge Lingán colegas del Museo de Historia Natural, quienes nos ayudaron con la identificación de las especies colectadas.

\section{Literatura citada}

Arana C. \& L. Salinas. 2003. Flora vascular de los Humedales de Chimbote, Perú. Rev peru Biol, 10(2): 221-224.

BOLFOR, B. Mostacero \& T.S. Frederickstein. 2000. Manual de Métodos Básicos de muestreo y análisis en Ecología Vegetal. BOLFOR. Editora El País, Santa Cruz, Bolivia. Pp. 16-17.

Bridson D. \& L. Forman. 1992. Herbarium Handbook. Royal Botanical Gardens, Kew. 303pp

Cano A., M.I. La Torre, B. León, et al. 1998. Estudio Comparativo de la Flora vascular de los Principales Humedales de las Zona Costera del Departamento de Lima, Perú. En: A. Cano y K.R. Young (Eds.) Los Pantanos de Villa: Biología y Conservación. Serie de Divulgación $N^{\circ} 11$, Museo de Historia Natural, Universidad Nacional Mayor de San Marcos, Lima: 181-190.

Clarkson B.R., B. Sorrell, P. Reeves, P. et al. 2004. Handbook for Monitoring Wetland Conditions. Ministry for the Environment Sustainable Management Fund Project (5105). (http://www.landcareresearch.co.nz/research/biocons/ restoration/docs/handbook2004.pdf) (Acceso: 1/nov/2009)
Cooke J., A. Cooper \& N. Clunie. 1990. Changes in the Water, Soil, and Vegetation of a Wetland after a decade of Receiving a Sewage Effluent. New Zealand Journal of Ecology. Vol. 14. :37-47.

Hammer O., D.A.T. Harper \& P.D. Ryan. 2001. PAST: Paleontological Statistics Software package for education and data analysis. Paleontologia Electrónica 4(1): 9pp.

León B. \& K.R. Young. 1996. Aquatic plants of Perú: diversity, distribution and conservation. Biodiversity and Conservation 5: 1169-1190.

León B. 1993. Catálogo anotado de las fanerógamas acuáticas del Perú. En: F. Kahn, B. León \& K.R. Young (eds.), Las Plantas Vasculares en las Aguas Continentales del Perú. Travaux de l'Institut Francais d'Etudes Andines Tomo 75. IFEA (Institut Francais d'Etudes Andines), Lima-Peru. $357 \mathrm{pp}$.

León B., A. Cano \& K.R. Young. 1995. La flora vascular de los Pantanos de Villa, Lima, Perú: Adiciones y guía para las especies comunes. Publicaciones del Museo de Historia Natural-UNMSM. (B) 38: 1-39.

León B., K.R. Young \& A. Cano. 1997. Fitogeografía y Conservación de la Costa Central del Perú. En: R. Valencia \& H. Balslev (Eds) Estudios Sobre Diversidad y Ecología de plantas (Memorias del II Congreso Ecuatoriano de Botánica) Pontificia Universidad católica de Ecuador, Quito. Pp.129-142.

León B., K.R. Young \& A. Cano. 1998. Uso Actual de la Flora y Vegetación en los Humedales de la Costa Central del Perú. En: A. Cano y K.R. Young (Eds.) Los Pantanos de Villa: Biología y Conservación. Serie de Divulgación $\mathrm{N}^{\circ} 11$, Museo de Historia Natural, Universidad Nacional Mayor de San Marcos, Lima:181-204.

Mitsch W.J. \& J.G. Gosselink. 1993. Wetlands, 2nd Edition. Van Nostrand Reinhold Co., New York, NY, USA. 722 pp.

Montoya H. 1998. La Diversidad de Algas y sus Roles en el Ecosistema. En: A. Cano y K.R. Young (Eds.) Los Pantanos de Villa: Biología y Conservación. Serie de Divulgación $\mathrm{N}^{\mathrm{o}} 11$, Museo de Historia Natural, Universidad Nacional Mayor de San Marcos, Lima: 21-40.

Sagástegui A. 1973. Manual de las Malezas de la Costa Norperuana. Primera Edición. Universidad Nacional de Trujillo. Trujillo, Perú. 480 pp.

Tovar O. 1993. Las Gramíneas (Poáceas) del Perú. Ruizia, tomo 13, Madrid. $481 \mathrm{pp}$.

USDA. 2000. Activity Report: Kika de la Garza Plant Material Center, Kingsville, Texas. United States Department of Agriculture Natural Resources Conservation Service. Plant Material Program.

Wittaker R.H. 1975. Communities and ecosystems. Macmillan Publishing Co., Inc. Edition 2a. 385 pp

Young K.R: 1998. El Ecosistema. En: A. Cano y K. Young (Eds.) Los Pantanos de Villa: Biología y Conservación. Serie de Divulgación, Museo de Historia Natural-UNMSM. 11:3-20. 
Apéndice 1. Lista de familias y especies de la Flora Vascular del Humedal Santa Rosa, indicando la forma de crecimiento (FC): $\mathrm{H}$ : Hierba; A: Arbusto. Dentro de las hierbas acuáticas se especifica si son Flotantes (FL); Emergente (E); Sumergida (S). Igualmente se especifica si corresponde a una especie Introducida o Nativa ( $\circ$ N) en humedales.

\begin{tabular}{|c|c|c|c|}
\hline & ESPECIE & FC & $\mathrm{I} / \mathrm{N}$ \\
\hline & ADIANTACEAE & & \\
\hline \multirow[t]{2}{*}{1.} & Adiantum digitatum Hook. & $\mathrm{H}$ & I \\
\hline & AIZOACEAE & & \\
\hline 2. & Sesuvium portulacastrum (L.) L. & $\mathrm{H}$ & $\mathrm{N}$ \\
\hline \multirow[t]{2}{*}{3.} & Trianthema portulacastrum L. & $\mathrm{H}$ & I \\
\hline & APIACEAE & & \\
\hline 4. & Hydrocotyle bonariensis Lam. & $\mathrm{H}$ & $\mathrm{N}$ \\
\hline 5. & Hydrocotyle ranunculoides L.f. & $\mathrm{H}$ & $\mathrm{N}$ \\
\hline \multirow[t]{2}{*}{6.} & Cyclospermun laciniatum (DC.) Constance & $\mathrm{H}$ & I \\
\hline & ARACEAE & & \\
\hline 7. & Pistia stratiotes L. & $\mathrm{H}(\mathrm{FL})$ & $\mathrm{N}$ \\
\hline \multirow[t]{2}{*}{8.} & Colocasia esculenta (L.) Schott & $\mathrm{H}$ & I \\
\hline & ASTERACEAE & & \\
\hline 9. & Aster exilis Elliott & $\mathrm{H}$ & I \\
\hline 10. & Ambrosia artemisiifolia $\mathrm{L}$. & $\mathrm{H}$ & I \\
\hline 11. & Conyza bonariensis L. & $\mathrm{H}$ & I \\
\hline 12. & Eclipta prostrata (L.) L. & $\mathrm{H}$ & I \\
\hline 13. & Tessaria integrifolia Ruiz \& Pav. & A & $\mathrm{N}$ \\
\hline \multirow[t]{2}{*}{14.} & Enydra sessilifolia (Ruiz \& Pav.) Cabrera & $\mathrm{H}$ & $\mathrm{N}$ \\
\hline & AZOLLACEAE & & \\
\hline \multirow[t]{2}{*}{15.} & Azolla filiculoides Lam. & $\mathrm{H}(\mathrm{FL})$ & $\mathrm{N}$ \\
\hline & BORAGINACEAE & & \\
\hline \multirow[t]{2}{*}{16.} & Heliotropium curassavicum L. & $\mathrm{H}$ & $\mathrm{N}$ \\
\hline & BRASSICACEAE & & \\
\hline \multirow[t]{2}{*}{17.} & Nasturtium officinalle R. Br. & $\mathrm{H}$ & I \\
\hline & CHENOPODIACEAE & & \\
\hline 18. & Chenopodium ambrossoides L. & $\mathrm{H}$ & I \\
\hline 19. & Chenopodium murale $\mathrm{L}$. & $\mathrm{H}$ & $\mathrm{N}$ \\
\hline \multirow[t]{2}{*}{20.} & Sarcocornia neei (Lag.) M.A. Alonso \& M.B. Crespo. & $\mathrm{H}$ & $\mathrm{N}$ \\
\hline & CONMELINACEAE & & \\
\hline \multirow[t]{2}{*}{21.} & Commelina fasciculata Ruiz \& Pav. & $\mathrm{H}$ & I \\
\hline & CYPERACEAE & & \\
\hline 22. & Cyperus articulatus L. & $\mathrm{H}$ & $\mathrm{N}$ \\
\hline 23. & Cyperus laevigatus L. & $\mathrm{H}$ & $\mathrm{N}$ \\
\hline 24. & Torulinium odoratum (L.) S.S. Hooper & $\mathrm{H}$ & I \\
\hline 25. & Eleocharis geniculata (L.) Roem. \& Schult. & $\mathrm{H}$ & $\mathrm{N}$ \\
\hline 26. & Killinga brevifolia Rottb. & $\mathrm{H}$ & I \\
\hline \multirow[t]{2}{*}{27.} & Schoenoplectus americanus (Pers.) Volkart ex Schinz \& R. Keller & $\mathrm{H}$ & $\mathrm{N}$ \\
\hline & FABACEAE & & \\
\hline \multirow[t]{2}{*}{28.} & Medicago lupulina $\mathrm{L}$. & $\mathrm{H}$ & I \\
\hline & HALORAGIDACEAE & & \\
\hline \multirow[t]{2}{*}{29.} & Myriophyllum aquaticum (Vell.) Verdc. & $\mathrm{H}(\mathrm{E})$ & $\mathrm{N}$ \\
\hline & LAMIACEAE & & \\
\hline 30. & Mentha aff. viridis & $\mathrm{H}$ & I \\
\hline \multirow[t]{2}{*}{31.} & Mentha spicata (L.) & $\mathrm{H}$ & I \\
\hline & LEMNACEAE & & \\
\hline 32. & Lemna gibba L. & $\mathrm{H}(\mathrm{FL})$ & $\mathrm{N}$ \\
\hline 33. & Lemna minuta Kunth. & $\mathrm{H}(\mathrm{FL})$ & $\mathrm{N}$ \\
\hline \multirow[t]{2}{*}{34.} & Wolffia columbiana H. Karst. & $\mathrm{H}(\mathrm{FL})$ & $\mathrm{N}$ \\
\hline & ONAGRACEAE & & \\
\hline 35. & Ludwigia peploides (Kunth) P.H. Raven & $\mathrm{H}$ & $\mathrm{N}$ \\
\hline 36. & Ludwigia peruviana (L.) Hara & $\mathrm{H}$ & $\mathrm{N}$ \\
\hline \multirow[t]{2}{*}{37.} & Ludwigia octovalvis (Jacq.) P.H. Raven & $\mathrm{H}$ & $\mathrm{N}$ \\
\hline & OXALIDACEAE & & \\
\hline \multirow[t]{2}{*}{38.} & Oxalis corniculata $\mathrm{L}$. & $\mathrm{H}$ & I \\
\hline & PLANTAGINACEAE & & \\
\hline \multirow[t]{2}{*}{39.} & Plantago major L. & $\mathrm{H}$ & I \\
\hline & & & Continúa. \\
\hline
\end{tabular}


Apéndice 1.

\section{POACEAE}

40. Arundo donax L.

41. Cynodon dactylon (L.) Pers.

42. Distichlis spicata (L.) Greene

43. Echinochloa cruspavonis (Kunth) Schult.

44. Echinochloa oryzoides (Ard.) Fritsch

45. Eleusine indica (L.) Gaertn.

46. Eriochloa procera (Retz.)

47. Leptochloa uninervia (J. Presl) Hitchc. \& Chase

48. Paspalidium geminatum (Forssk.) Stapf

49. Paspalum conjugatum P.J. Bergius

50. Paspalum lividum Trin. ex Schltdl.

51. Paspalum vaginatum Swartz.

52. Polypogon elongatus Kunth

53. Polypogon semiverticillatus (Forssk.) Hyl.

54. Setaria geniculata (Willd.) P. Beauv.

55. Sporobolus virginicus (L.) Kunth POLYGONACEAE

56. Rumex obtusifolius L.

57. Rumex crispus $\mathrm{L}$.

58. Polygonum hydropiperoides Michx. PONTEDERIACEAE

59. Eichhornia crassipes (Mart.) Solms_Laubach SCROPHULARIACEAE

60. Bacopa monnieri (L.) Wettst. SOLANACEAE

61. Solanum americanum Mill.

62. Acnistus arborescens (L.) Schltdl. THYPHACEAE

63. Typha domingensis Pers. VERBENACEAE

64. Lippia nodiflora (L.) Michx

65. Verbena bonariensis L. ZANNICHELIACEAE

66. Zannichellia palustris $\mathrm{L}$.

$\begin{array}{cc}\text { A } & \text { I } \\ \text { H } & \text { I } \\ \text { H } & \text { N } \\ \text { H } & \text { I } \\ \text { H } & \text { I } \\ \text { H } & \text { I } \\ \text { H } & \text { I } \\ \text { H } & \text { I } \\ \text { H } & \text { N } \\ \text { H } & \text { I } \\ \text { H } & \text { N } \\ \text { H } & \text { N } \\ \text { H } & \text { N } \\ \text { H } & \text { I } \\ \text { H } & \text { I } \\ \text { H } & \text { N } \\ & \\ \text { H } & \text { I } \\ \text { H } & \text { I } \\ \text { H } & \text { N }\end{array}$

$\mathrm{H}(\mathrm{FL}) \quad \mathrm{N}$

$\mathrm{H} \quad \mathrm{N}$

$\mathrm{H} \quad \mathrm{I}$

A I

$\mathrm{H}(\mathrm{E}) \quad \mathrm{N}$

H N

$\mathrm{H} \quad \mathrm{I}$

H(S) N

